\title{
Lesões perinatais em bovinos na intoxicação experimental por Ateleia glazioviana (Leg.Papilionoideae) ${ }^{1}$
}

\author{
Maria del Carmen García y Santos ${ }^{2}$, Ana Lucia Schild ${ }^{3 *}$, Severo Sales de Barros ${ }^{4}$, \\ Franklin Riet-Correa ${ }^{5}$, Fabiana Elias ${ }^{6}$ e Adriano T. Ramos ${ }^{6}$
}

\begin{abstract}
García y Santos M.C., Schild A.L., Barros S.S., Riet-Correa F., Elias F. \& Ramos A.T. 2004. [Perinatal lesions in cattle experimentally poisoned by Ateleia glazioviana (Leg. Papilionoideae).] Lesões perinatais em bovinos na intoxicação experimental por Ateleia glazioviana (Leg.Papilionoideae).] Pesquisa Veterinária Brasileira 24(3):178-184. Laboratório Regional de Diagnóstico, Faculdade de Veterinária, UFPel, Cx.Postal 354, Pelotas, RS 96010-900, Brazil. E-mail: alschild@terra.com.br

Leaves of Ateleia glaziovian Baill., dried in a $100^{\circ} \mathrm{C}$ oven for $16-20$ hours, were given to seven crossbred cows, always as bolus. Two of them received $9 \mathrm{~g} / \mathrm{kg}$ at 4 months of pregnancy. Three cows in the 8th month of pregnancy received daily doses of $1-2 \mathrm{~g} / \mathrm{kg}$ of the leaves, until a total amount of 10,21 and $28 \mathrm{~g} / \mathrm{kg} / \mathrm{bw}$ was reached. Two 8-month-pregnant cows were fed 15.5 and 18 $\mathrm{g} / \mathrm{kg}$ of the dried leaves. Two 4-month-pregnant cows were fed $35 \mathrm{~g} / \mathrm{kg}$ of green leaves of $A$. glazioviana. The cow treated with $21 \mathrm{~g} / \mathrm{kg}$ of the dry plant material showed clinical signs of poisoning and delivered a stillborn calf. No clinical signs were observed in the other cows. The calves from cows that received $9,15.5$ and $28 \mathrm{~g} / \mathrm{kg}$ of the dried leaves showed weakness and suckling difficulties, and were killed. Whitish areas and thickening of the right ventricle wall of the heart were observed in the stillborn calf. Thickening of the wall of the right ventricle was also observed in the calf delivered by the cow treated with $28 \mathrm{~g} / \mathrm{kg}$ of dry plant. The other calves had no gross lesions. The histological changes in all necropsied calves were characterized by tumefaction and vacuolization of muscle fibers and proliferation of fibrous tissue, similar as occurred in spontaneous cases of fibrosis of the heart muscle in cattle poisoned by A. glazioviana. PAS stained slides revealed positive granules in the cardiomyocytes more evident than those of the control calf. The stillborn calf had mild spongiosis of the white matter of the cerebellum, thalamus and rostral colliculi. The ultrastructural pathology revealed cardiac fibers with large glycogen storage within myofibril bundles, which showed loss of bundles and disappearance of whole sarcomers. Mild glycogen storage was observed in a control calf.
\end{abstract}

INDEX TERMS: Poisonous plants, plant poisoning, Ateleia glazioviana, Leguminosae Papilionoideae, abortion, cardiac fibrosis, glycogenosis.

\footnotetext{
${ }^{1}$ Recebido em 25 de maio de 2004.

Aceito para publicação em 28 de junho de 2004 .

Trabalho foi financiado pela FAPERGS, CNPq-PRONEX nº 661069/1997.

${ }^{2}$ Depto Patología, Área de Toxicología de la Facultad de Veterinária, Universidad de la República, Uruguay.

${ }^{3}$ Laboratório Regional de Diagnóstico, Faculdade de Veterinária, Universidade Federal de Pelotas (UFPel), 96010-900 Pelotas, RS. *Autor para correspondência. E-mail: alschild@terra.com.br

${ }^{4}$ Professor Visitante, Depto Patologia, Faculdade de Veterinária, UFPel.

${ }^{5}$ Centro de Saúde e Tecnologia Rural, Faculdade de Veterinária, UFCG, 58700-000 Patos, PB.

${ }^{6}$ Alunos do Curso de Pós-Graduação, Faculdade de Veterinária , UFPel.
}

RESUMO.- Folhas de Ateleia glazioviana Baill., dessecadas em estufa a $100^{\circ} \mathrm{C}$ por 16 a 20 horas, foram administradas a sete vacas sem raça definida. Duas vacas receberam $9 \mathrm{~g} / \mathrm{kg}$ da planta seca aos 4 meses de gestação em dose única. Três vacas no $8^{\circ}$ mês de gestação, receberam doses diárias de 1 a $2 \mathrm{~g} / \mathrm{kg}$ da planta seca, perfazendo totais de 10, 21 e $28 \mathrm{~g} / \mathrm{kg}$. Duas outras, também no $8^{\circ}$ mês de gestação, receberam a planta seca em dose única 15,5 e $18 \mathrm{~g} / \mathrm{kg}$. Duas vacas, no $4^{\circ}$ mês de gestação, receberam $35 \mathrm{~g} / \mathrm{kg}$ da planta verde, em dose única. A vaca que recebeu $21 \mathrm{~g} / \mathrm{kg}$ da planta seca foi a única a apresentar sinais clínicos de intoxicação e o bezerro nasceu morto. As demais vacas não apresentaram sinais clínicos. Os bezerros das vacas que receberam a 
planta seca nas doses de 9, 15,5 e $28 \mathrm{~g} / \mathrm{kg}$, apresentaram sinais clínicos de debilidade, dificuldade de manter-se em pé e mamar e foram sacrificados. Macroscopicamente, no bezerro que nasceu morto, foram observados áreas esbranquiçadas e espessamento da parede do ventrículo cardíaco direito. Os demais bezerros que nasceram fracos foram sacrificados e não apresentaram lesões macroscópicas, com exceção do bezerro da vaca que recebeu a planta seca, na dose total de $28 \mathrm{~g} / \mathrm{kg}$, que apresentou, também, espessamento da parede do ventrículo direito. As alterações histológicas do bezerro que nasceu morto e dos que foram sacrificados por apresentarem sinais clínicos de debilidade caracterizaram-se por tumefação e vacuolização de feixes de fibras musculares e proliferação de tecido fibroso entre os cardiomiócitos. Essas lesões são semelhantes às observadas em casos espontâneos da intoxicação. Os cardiomiócitos mostraram reação PAS positiva no sarcoplasma, mais acentuada do que no animal controle. No bezerro que nasceu morto foi observado, também, espongiose discreta na substância branca do cerebelo, colículos rostrais e tálamo. 0 estudo ultra-estrutural do coração revelou fibras cardíacas com acúmulos de gliocogênio, situados entre feixes de miofibrilas, os quais mostravam perda de miofilamentos e desaparecimento de sarcômeros inteiros. No animal controle a presença de glicogênio foi substancialmente menor do que nos animais intoxicados.

TERMOS DE INDEXAÇÃO: Plantas tóxicas, intoxicação por plantas, Ateleia glazioviana, Leguminosae Papilionoideae, aborto, fibrose cardíaca, glicogenose.

\section{INTRODUÇÃO}

Ateleia glazioviana e Tetrapterys spp (T. acutifolia e T. multiglandulosa) são plantas conhecidas no Brasil por causar, em bovinos, sinais nervosos, abortos e fibrose cardíaca manifestada por morte súbita ou sinais de insuficiência cardíaca congestiva (Tokarnia et al. 2000).

Ateleia glazioviana é uma das mais importantes plantas tóxicas do noroeste do Rio Grande do Sul e oeste de Santa Catarina. A intoxicação espontânea tem sido descrita em bovinos causando diferentes quadros clínicos, dependendo da quantidade e da forma como a planta é ingerida. A intoxicação espontânea em bovinos causa nascimento de animais fracos que morrem no período neonatal, abortos (Gava 1993), e fibrose do miocárdio, com morte súbita ou insuficiência cardíaca congestiva e sinais clínicos nervosos, (Gava 2001, Gava \& Barros 2001). Recentemente, foi diagnosticado um surto espontâneo de intoxicação pela planta em ovinos (Gava et al. 2003). A prevalência de aborto causado pela intoxicação espontânea por $A$. glazioviana em vacas a pastoreio pode variar entre 10 e $40 \%$, podendo em alguns surtos chegar a $100 \%$. A morbidade em casos de insuficiência cardíaca congestiva varia de 10 a $60 \%$ e a letalidade é próxima de 100\% (Gava \& Barros 2001).

Miocardiopatia, sinais clínicos nervosos e abortos ou nascimento de animais fracos têm sido reproduzidos experimentalmente em bovinos, pela administração de $A$. glazioviana verde ou dessecada, (Gava et al. 2001, Stolf et al. 1994). A forma cardíaca da enfermidade e sinais clínicos nervosos foram reproduzidos experimentalmente também em ovinos (Stigger et al. 2001, Leite et al.
2002). O princípio ativo cardiotóxico de A. glazioviana é estável a $100^{\circ} \mathrm{C}$ e ratos, camundongos, coelhos e cobaios não são suscetíveis ao efeito cardiotóxico da planta (Leite et al. 2002). O quadro nervoso da enfermidade, denominado forma letárgica, é caracterizada por marcada depressão, marcha incoordenada e batida contra objetos.

As lesões histológicas nos casos de morte súbita caracterizam-se por marcada degeneração e necrose dos miócitos e fibrose do miocárdio. Os animais que desenvolvem insuficiência cardíaca congestiva apresentam, além das lesões cardíacas, congestão passiva crônica do fígado e edemas generalizados. Em alguns casos observa-se, também, degeneração esponjosa no sistema nervoso. Quando o quadro clínico manifesta-se com sinais clínicos nervosos microscopicamente os animais apresentam degeneração esponjosa localizada do sistema nervoso central, principalmente, na substância branca da medula cerebelar, pedúnculos cerebelares, ponte e medula oblonga. (Gava \& Barros 2001, Gava et al. 2001).

A intoxicação por Tetrapterys spp causa sinais clínicos relacionados a insuficiência cardíaca, como veia jugular ingurgitada, pulso venoso positivo, edema da região da barbela e arritmia cardíaca. A evolução é subaguda a crônica, podendo ocorrer excepcionalmente "morte súbita" quando os animais são forçados a se movimentarem. Outros sinais clínicos observados são dificuldade de locomoção, lerdeza, fraqueza, anorexia, tremores musculares, leve dispnéia e fezes ressequidas. Ocorrem, também, abortos e nascimento de bezerros fracos que logo morrem (Tokarnia et al. 1989; Tokarnia et al. 2000).

O quadro clínico de cardiomiopatia causado por Ateleia glazioviana e Tetrapterys spp tem sido observado, também, no sul da África, em ruminantes que consomem plantas da família Rubiaceae (Pachystigma spp, Pavetta spp e Fadogia monticola), que também causam fibrose cardíaca. Fourie (1995) comprovou que o precipitado etanólico de Pavetta harborii causa os mesmos sinais clínicos e lesões, observados na intoxicação natural por A. glazioviana. Uma substância denominada pavetamine contida no precipitado etanólico foi identificada como o princípio ativo (Schultz et al. 2001). A semelhança das manifestações clínicas e lesões causadas pelas plantas da família Rubiaceae presentes no sul da África como as causadas por Ateleia glazioviana e Tetrapterys spp sugere que estas últimas possam conter o mesmo princípio ativo. Esta semelhança é sugerida, também, pelo fato de que tanto o princípio ativo das plantas do sul da África quanto o princípio ativo de $A$. glazioviana não afetam animais de laboratório quando administradas pela via oral e são termoestáveis a $100^{\circ} \mathrm{C}$ (Leite et al. 2002).

Os objetivos deste trabalho foram: determinar se o princípio ativo de $A$. glazioviana atravessa a barreira placentária causando lesões neonatais em bezerros de vacas que ingerem a planta durante a gestação; determinar se o princípio ativo da planta, estável a $100^{\circ} \mathrm{C}$, que causa fibrose cardíaca e espongiose do sistema nervoso é, também, responsável pelos abortos ocasionados pela planta; e estudar as lesões histológicas e ultra-estruturais observadas nos fetos e/ou animais nascidos fracos.

\section{MATERIAL E MÉTODOS}

Para determinar se o princípio ativo de Ateleia glazioviana que causa fibrose cardíaca atravessa a barreira placentária e causa lesões e 
morte fetal, foram utilizadas cinco vacas (numeradas 1 a 5 ) sem raça definida prenhes no $8^{\circ}$ mês de gestação, que receberam a planta seca à sombra e moída, sendo posteriormente passada em estufa a $100^{\circ} \mathrm{C}$ por 16 a 20 horas. Vaca 4 recebeu $1 \mathrm{~g} / \mathrm{kg}(\mathrm{g} / \mathrm{kg}$ de peso corporal) de planta durante 10 dias. Vaca 1 recebeu $1 \mathrm{~g} / \mathrm{kg}$ de planta seca por dia durante 10 dias e $2 \mathrm{~g} / \mathrm{kg}$ por mais 5 dias. Em razão de apresentar sinais clínicos a administração da planta foi suspensa por 3 dias e após esse intervalo, por ter melhorado, voltou a receber $1 \mathrm{~g} / \mathrm{kg}$ durante um dia, perfazendo um total de $21 \mathrm{~g} / \mathrm{kg}$ de planta. Estes animais recebiam a planta seca, misturada a ração comercial para bovinos que era administrada em quantidade equivalente a $1 \%$ do peso corporal. Vaca 3 recebeu $1 \mathrm{~g} / \mathrm{kg}$ de planta seca durante 8 dias, por sonda ruminal, e após intervalo de 10 dias recebeu $2 \mathrm{~g} / \mathrm{kg}$ durante mais 10 dias, perfazendo um total de $28 \mathrm{~g} / \mathrm{kg}$. Vacas 2 e 5 receberam doses únicas de 15,5 e $18 \mathrm{~g} / \mathrm{kg}$ de planta seca por sonda ruminal.

Quatro vacas sem raça definida prenhes no $4^{\circ}$ mês de gestação foram utilizadas no experimento, duas delas (Vacas 6 e 7) receberam 9 $\mathrm{g} / \mathrm{kg}$ de planta seca em dose única. A planta moída foi passada em estufa a $100^{\circ} \mathrm{C}$ por 16 a 20 horas, sendo administrada misturada à ração comercial que era administrada em quantidade equivalente a $2 \%$ do peso corporal de cada animal. Vacas 8 e 9 receberam, em dose única, $35 \mathrm{~g} / \mathrm{kg}$, da planta verde, triturada e misturada com forragem verde (capim-elefante). Os dados sobre os animais, quantidade de planta administrada e período de administração são apresentados no Quadro 1.

A. glazioviana administrada às Vacas 1,2 e 4 foi coletada no município de Campos Novos, SC, no mês de setembro de 2001. A planta administrada às Vacas $3,5,6$ e 7 era proveniente do município de Ijuí, RS, tendo sido coletada em fevereiro de 2002. Para as Vacas 8 e 9, a planta administrada foi coletada, em maio de 2002, de duas árvores plantadas na Faculdade de Veterinária da UFPel, município de Capão do Leão.

As funções cardíacas dos nove animais foram monitoradas por exame clínico diário sendo avaliados as frequiências cardíaca e respiratória, temperatura retal, movimentos ruminais e modificações do comportamento, postura e movimentação. Os fetos foram monitorados diariamente por palpação retal e semanalmente por ultra-sonografia. Os bezerros que nasceram com sinais clínicos de

Quadro 1. Peso dos animais, período de gestação, quantidade de planta diária e dose total de Ateleia glazioviana administrada a 9 vacas

\begin{tabular}{ccccccc}
\hline $\begin{array}{c}\text { Vaca } \\
\text { no. }\end{array}$ & $\begin{array}{c}\text { Peso } \\
(\mathrm{kg})\end{array}$ & $\begin{array}{c}\text { Período de } \\
\text { gestação } \\
\text { (meses) }\end{array}$ & Planta & $\begin{array}{c}\text { Dose diária de } \\
\text { planta }(\mathrm{g} / \mathrm{kg})\end{array}$ & $\begin{array}{c}\text { Período } \\
(\text { dias })\end{array}$ & $\begin{array}{c}\text { Total de } \\
\text { planta }(\mathrm{g} / \mathrm{kg})\end{array}$ \\
\hline $1^{\mathrm{a}}$ & 343 & 8 & Seca & $1-2$ & 16 & 21 \\
2 & 258 & 8 & Seca $^{\mathrm{b}}$ & 15,5 & 1 & 15,5 \\
$3^{\mathrm{c}}$ & 375 & 8 & Seca $^{\mathrm{b}}$ & $1-2$ & 18 & 28 \\
4 & 310 & 8 & Seca $^{2}$ & 1 & 10 & 10 \\
5 & 360 & 8 & Seca & 18 & 1 & 18 \\
6 & 337 & 4 & Seca & 9 & 1 & 9 \\
7 & 341 & 4 & Seca & 9 & 1 & 9 \\
8 & 520 & 4 & Verde & 35 & 1 & 35 \\
9 & 370 & 4 & Verde & 35 & 1 & 35 \\
10 & Controle & & & & & \\
\hline
\end{tabular}

a Vaca 1 recebeu $1 \mathrm{~g} / \mathrm{kg}$ durante 10 dias e $2 \mathrm{~g} / \mathrm{kg}$ por 5 dias, apresentou sinais clínicos sendo suspensa administração por 3 dias e após recebeu 1 $\mathrm{g} / \mathrm{kg}$ por um dia.

b Planta administrada por sonda ruminal.

c Vaca 3 recebeu durante 8 dias $1 \mathrm{~g} / \mathrm{kg}$, apresentou lesões esofágicas em conseqüência da passagem da sonda e a administração foi suspensa por 10 dias, quando voltou a receber $2 \mathrm{~g} / \mathrm{kg}$ por 10 dias. debilidade, dificuldade de manter-se em pé e mamar foram sacrificados e necropsiados. Fragmentos de órgãos das cavidades torácica e abdominal e encéfalo foram fixados em formol tamponado a $10 \%$ e processados rotineiramente para histologia e corados pela hematoxilina-eosina (HE). Fragmentos de músculo estriado, coração, fígado, rim, pulmão e encéfalo foram corados pela técnica do ácido periódico de Schiff(PAS). Cortes do miocárdio foram corados pelo PAS tendo sido previamente tratados pela diastase salivar por 20 minutos. Bezerro 20 da Vaca 10 (controle) que permanecia no mesmo potreiro dos animais experimentais e recebia ração comercial em quantidade equivalente a $1 \%$ do peso corporal, foi sacrificado e necropsiado para ser utilizado como bezerro controle.

Para estudo ultra-estrutural, fragmentos do miocárdio e sistema nervoso central foram fixados em solução de glutaraldeído $2 \%$ e paraformaldeído $2 \%$ em tampão cacodilato de sódio, desidratados em uma série crescente de etanóis e incluídos em Epon. Os cortes semi-finos foram corados por azul de metileno. Em áreas selecionadas dos blocos foram feitos cortes ultra-finos contrastados por acetato de uranila e citrato de chumbo e observados em microscópio eletrônico de transmissão.

\section{RESULTADOS}

\section{Sinais clínicos}

Das vacas que receberam a planta seca, a única que apresentou sinais clínicos de intoxicação foi a Vaca 1, que após a ingestão de $21 \mathrm{~g}$ de planta por $\mathrm{kg}$ de peso vivo apresentou letargia, depressão e incoordenação motora. Houve regressão desses sinais após a suspensão da administração da planta por 3 dias. Este animal, dois dias após ter parado de ingerir a planta, pariu um bezerro macho (Bezerro 11), nascido a termo, morto durante o parto que apresentava na necropsia discreto edema subcutâneo na cabeça e no pescoço e aeração dos bordos pulmonares. Todas as demais vacas do experimento, tanto as que receberam planta verde como as que receberam a planta dessecada tiveram partos normais, dentro do tempo esperado para a espécie bovina. A avaliação clínica diária não revelou qualquer alteração durante o período experimental, com exceção da Vaca 1 que apresentou sinais clínicos de letargia, razão pela qual a administração da planta foi suspensa por 3 dias. Os Bezerros 12, 13 e 16 das Vacas 2, 3 e 6, respectivamente, nasceram com sinais clínicos de debilidade e dificuldade para manter-se em estação (Fig. 1). No Bezerro 16 estes sinais eram discretos (Quadro 2). Bezerro 15 , que foi sacrificado para estudo histológico, não apresentou sinais clínicos. Os Bezerros 18 e 19 das vacas 8 e 9 , respectivamente, o Bezerro 14 da Vaca 4 e o Bezerro 17 da vaca 7 nasceram clinicamente normais e foram observados por 3 meses, sem que apresentassem sinais de alteração cardíaca ou do sistema nervoso central (Quadro 2).

\section{Patologia macroscópica e histológica}

Foram sacrificados e necropsiados os Bezerros 12, 13, 15 e 16. Na necropsia do Bezerro 11, que nasceu morto, observaram-se edema da cabeça e do pescoço, pequena aeração nos bordos pulmonares e ausência de coágulo nos vasos umbilicais. O coração, ao corte, apresentava espessamento da parede do ventrículo direito e áreas esbranquiçadas, principalmente, nos vértices do septo interventricular. O Bezerro 13 apresentou, também, espessamento da parede do ventrículo direito. Os demais 
Quadro 2. Sinais clínicos e lesões macroscópicas e histológicas observadas nos bezerros das vacas que ingeriram Ateleia glazioviana

\begin{tabular}{|c|c|c|c|c|c|c|c|c|c|c|}
\hline \multirow{3}{*}{$\begin{array}{c}\text { Bezerro } \\
\text { no. }\end{array}$} & \multirow{3}{*}{$\begin{array}{l}\text { Vaca } \\
\text { no. }\end{array}$} & \multirow{3}{*}{$\begin{array}{l}\text { Período de } \\
\text { gestação }\end{array}$} & \multirow{3}{*}{$\begin{array}{l}\text { Total de } \\
\text { planta }\end{array}$} & \multirow{3}{*}{$\begin{array}{l}\text { Tempo de } \\
\text { ingestão }\end{array}$} & \multirow{3}{*}{$\begin{array}{l}\text { Sinais clínicos } \\
\text { de debilidade }\end{array}$} & \multirow{2}{*}{\multicolumn{2}{|c|}{$\begin{array}{c}\text { Alterações macroscó- } \\
\text { picas cardíacas }\end{array}$}} & \multicolumn{3}{|c|}{ Alterações histológicas cardíacas } \\
\hline & & & & & & & & \multicolumn{2}{|r|}{ HE } & \multirow{2}{*}{$\frac{\text { PAS }}{\text { Grânulos positivos }}$} \\
\hline & & & & & & Hipertrofia & Áreas claras & Fibrose & Vacuolização & \\
\hline 11 & 1 & 8 meses & $21 \mathrm{~g} / \mathrm{kg}$ & 16 dias & natimorto & $++++^{b}$ & +++ & +++ & ++ & ++ \\
\hline 12 & 2 & 8 meses & $15,5 \mathrm{~g} / \mathrm{kg}$ & 1 dia & ++++ & - & - & - & ++ & +++ \\
\hline 13 & 3 & 8 meses & $28 \mathrm{~g} / \mathrm{kg}$ & 18 dias & +++ & ++++ & - & ++ & +++ & ++++ \\
\hline 14 & 4 & 8 meses & $10 \mathrm{~g} / \mathrm{kg}$ & 10 dias & - & $\mathrm{NE}^{\mathrm{d}}$ & NE & NE & NE & NE \\
\hline 15 & 5 & 8 meses & $18 \mathrm{~g} / \mathrm{kg}$ & $1 \mathrm{dia}$ & - & - & - & ++ & - & ++ \\
\hline 16 & 6 & 4 meses & $9 \mathrm{~g} / \mathrm{kg}$ & $1 \mathrm{dia}$ & ++ & - & - & ++ & - & ++ \\
\hline 17 & 7 & 4 meses & $9 \mathrm{~g} / \mathrm{kg}$ & 1 dia & - & $\mathrm{NE}$ & $\mathrm{NE}$ & $\mathrm{NE}$ & $\mathrm{NE}$ & $\mathrm{NE}$ \\
\hline 18 & $8^{a}$ & 4 meses & $35 \mathrm{~g} / \mathrm{kg}$ & 1 dia & - & $\mathrm{NE}$ & $\mathrm{NE}$ & $\mathrm{NE}$ & NE & $\mathrm{NE}$ \\
\hline 19 & $9^{a}$ & 4 meses & $35 \mathrm{~g} / \mathrm{kg}$ & 1 dia & - & $\mathrm{NE}$ & $\mathrm{NE}$ & $\mathrm{NE}$ & $\mathrm{NE}$ & $\mathrm{NE}$ \\
\hline $20^{c}$ & 10 & 9 meses & Controle & & - & - & - & - & - & + \\
\hline
\end{tabular}

a Animais que receberam planta verde.

${ }^{\text {b} G r a u ~ d e ~ s i n a i s ~ c l i ́ n i c o s ~ e ~ a l t e r a c ̧ o ̃ e s ~ m a c r o s c o ́ p i c a s ~ e ~ h i s t o l o ́ g i c a s: ~}++++$ acentuados, +++ moderados, ++ discretos, + normal, ausente.

c Bezerro controle.

${ }^{\mathrm{d}} \mathrm{NE}=$ não examinado.

bezerros que foram necropsiados, não apresentaram lesões macroscópicas (Quadro 2).

As lesões histológicas observadas no Bezerro 11 caracterizaram-se por proliferação de tecido fibroso, envolvendo alguns feixes de fibras cardíacas (Fig. 2) e presença de macrófagos no interstício. Em algumas áreas observaram-se vacuolização de fi- bras musculares e presença de miofibras eosinofilicas. No Bezerro 13 foram observadas áreas focais com grupos de miofibras cardíacas tumefeitas e vacuolizadas, melhor evidenciadas quando cortadas transversalmente (Fig.3). Bezerro 12 apresentava discretas áreas de vacuolização de miócitos, melhor evidenciadas, também, nas fibras cortadas transversalmente. Pela coloração de

Fig. 1. Bezerro 2 nascido de vaca intoxicada por Ateleia glazioviana, apresentando sinais de debilidade e apatia.

Fig. 2. Miocárdio do Bezerro 1 apresentando tecido fibroso aumentado entre as fibras cardíacas. HE, obj. 20.

Fig. 3. Miocárdio do Bezerro 3 apresentando vacuolização dos cardiomiócitos. HE, obj. 20.
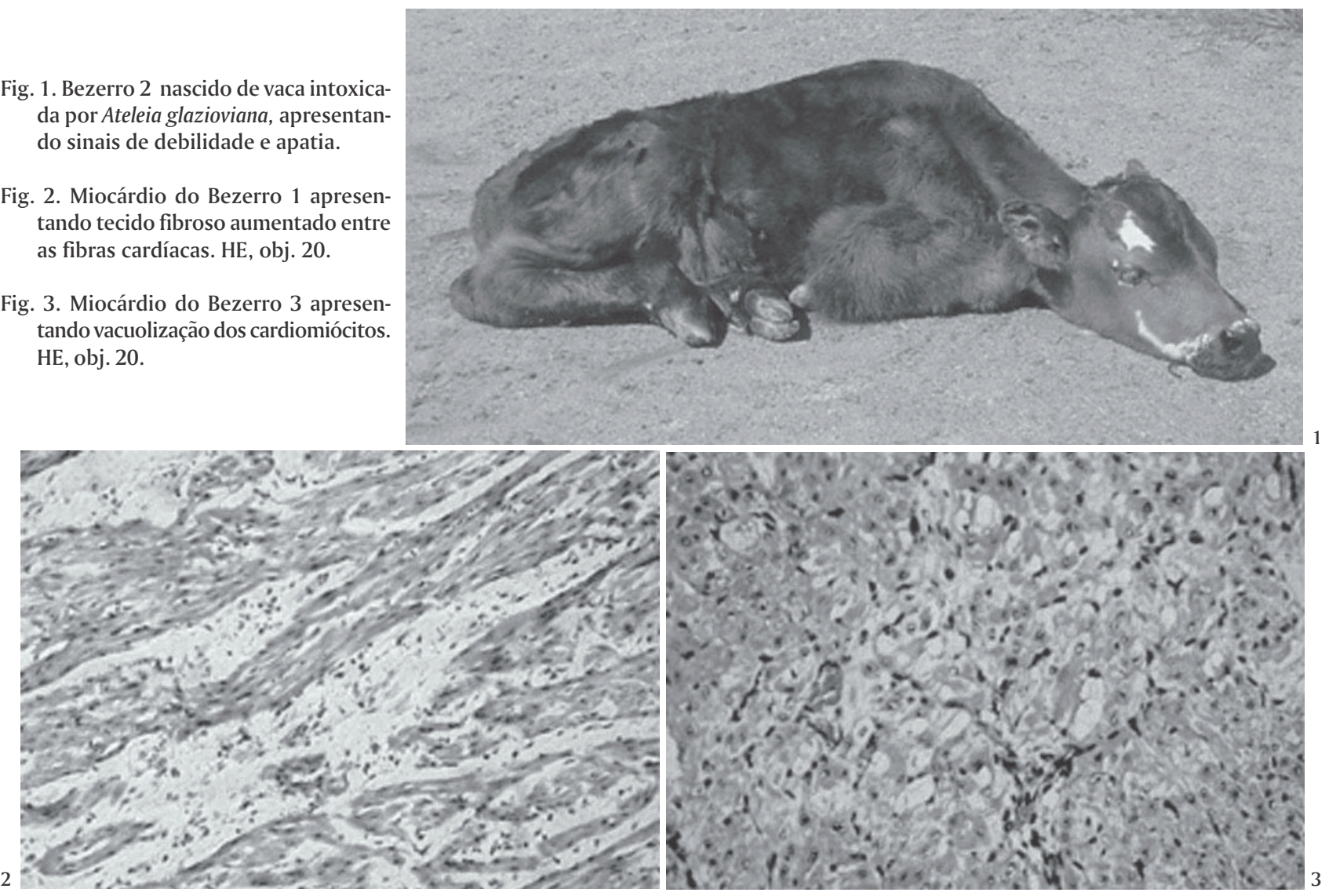
PAS evidenciou-se a presença de grânulos PAS positivos no miocárdio dos quatro bezerros experimentais e no controle. No Bezerro 13 a presença desses grânulos era acentuada e difusa, quando comparada ao animal testemunha; Bezerro 12 apresentava áreas focais com moderada presença de grânulos PAS positivos; nos Bezerros 11, 15 e 16 a presença dos grânulos era menos acentuada e se apresentava em áreas multifocais (Quadro 2); no Bezerro 20 (controle) a presença desses grânulos era sempre mais discreta do que nos animais experimentais. Nos fígados dos Bezerros 12, 13 e 16 havia vacuolização difusa dos hepatócitos. Pela coloração com PAS não se observou diferença na quantidade de grânulos positivos entre os animais experimentais e o Bezerro 20 (controle) no fígado, rins, pulmão e encéfalo. Os cortes tratados pela diastase salivar não exibiam grânulos PAS positivos tanto nos animais experimentais como no controle. No Bezerro 11 foi observada discreta vacuolização na substância branca das folhas cerebelares e na substância branca do tálamo e dos colículos rostrais. Nos demais bezerros, não foram observadas lesões do sistema nervoso. Nos demais órgãos, não foram observadas lesões macroscópicas ou histológicas.

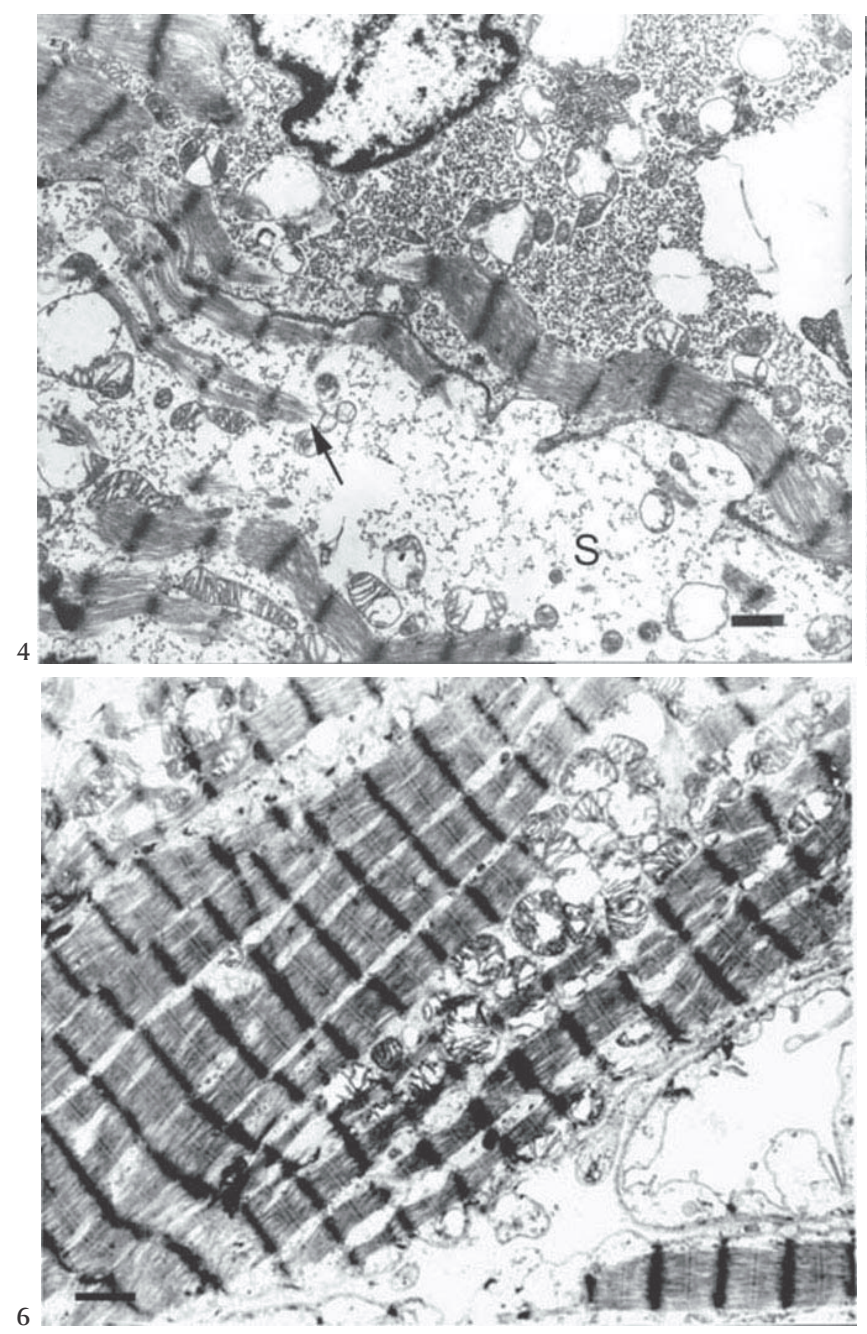

\section{Patologia ultra-estrutural}

A patologia ultra-estrutural do coração foi realizada nos Bezerros 13, 16 e 20. As fibras cardíacas exibiam acúmulos de gliocogênio, na forma monoparticulada, situados entre os feixes de miofibrilas (Fig.4), os quais mostravam perda de miofilamentos e desaparecimento de sarcômeros inteiros (Fig.4). As mitocôndrias estavam tumefeitas com distorção, encurtamento e desaparecimento das cristas. A matriz mitocondrial mostrava-se eletro-lúcida. Em várias áreas as fibras musculares exibiam acentuado edema do sarcoplasma com separação dos feixes de miofibrilas e formação de grandes áreas eletro-lúcidas (Fig.4). Edema intersticial moderado separando as miofibras foi observado em diversos pontos. $O$ retículo sarcoplásmico não exibia alterações notáveis, mas os sistemas T mostravam-se rompidos em alguns pontos. No animal controle a presença de glicogênio era sensivelmente menor do que nos animais intoxicados, mas as mitocôndrias exibiam alterações similares àquelas dos intoxicados (Fig. 5). Cardiomiócitos necróticos foram observados raramente, alguns deles em processo de fagocitose por macrófagos (Fig.6).

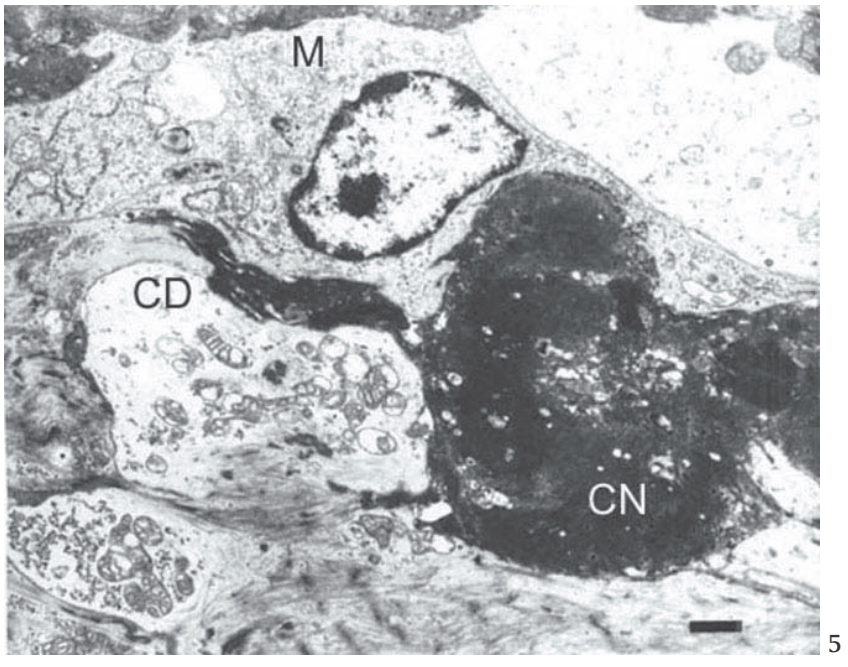

Fig. 4. Micrografia Eletrônica. Miocárdio do Bezerro 5. Cardiomiócitos com abundantes depósitos de glicogênio sob forma monoparticulada, distribuídos entre os feixes de miofibrilas que aparecem separados e com diminuição ou desaparecimento de sarcômeros (seta). As mitocôndrias estão tumefeitas com desorganização e ruptura das cristas. No cardiomiócito há acentuado edema do sarcoplasma (S). Barra $=1 \mathrm{~mm}$

Fig. 5. Micrografia Eletrônica. Miocárdio do bezerro controle. Cardiomiócito exibindo distribuição uniforme dos feixes de miofibrilas com preservação da integridade dos sarcômeros. Discretos depósitos de glicogênio entre os feixes de miofibrilas. Muitas mitocôndrias mostram-se tumefeitas com distorções das cristas. Barra $=2 \mathrm{~mm}$

Fig. 6. Micrografia Eletrônica. Miocárdio do Bezerro 5. Cardiomiócito necrótico $(\mathrm{CN})$ sendo fagocitado por macrófago $(\mathrm{M})$. Cardiomiócito em degeneração $(\mathrm{CD})$ com desorganização das miofibrilas, edema do sarcoplasma, depósitos de glicogênio e tumefação das mitocôndrias. Barra $=2 \mathrm{~mm}$ 


\section{DISCUSSÃO}

Os resultados deste experimento demonstram que o princípio ativo de Ateleia glazioviana atravessa a barreira placentária, quando a planta é administrada em dose única ou fracionada para vacas no final da gestação, causando lesões cardíacas nos bezerros que nascem com sinais clínicos de debilidade e dificuldade de manter-se em pé e mamar. A planta causa, também, espongiose no SNC, que foi observada no único bezerro deste experimento que nasceu morto. $\mathrm{O}$ fato de que não ocorreram abortos tanto com a planta seca quanto com a planta verde, administradas em doses semelhantes em vacas com 4 meses de gestação, não permitiu alcançar um dos objetivos deste trabalho: determinar se $o$ princípio ativo, termoestável a $100^{\circ} \mathrm{C}$, que causa lesões cardíacas e nervosas é o mesmo princípio ativo que causa aborto. No entanto, a morte durante o parto de um bezerro a termo, sem que tenha ocorrido parto distócico sugere que a planta seca a $100^{\circ} \mathrm{C}$ causa morte fetal e, portanto, pode causar abortos. Neste caso, os abortos ocorreriam em conseqüência de lesões causadas pela planta nos fetos; doses maiores causariam lesões mais graves e abortos, enquanto que doses menores causariam o nascimento de bezerros fracos. Este fato pode ser constatado em um trabalho anterior no qual foram observados sinais clínicos de debilidade e dificuldade de mamar e manter-se em pé em bezerros nascidos de três vacas que ingeriram 22,23 e 30 g/ $/ \mathrm{kg}$ de $A$. glazioviana fresca, enquanto que três vacas que ingeriram $35 \mathrm{~g} / \mathrm{kg}$ abortaram depois de apresentaram sinais clínicos de intoxicação (Stolf et al. 1994). Em nosso experimento a Vaca 1 cujo bezerro morreu durante o parto foi a única que apresentou sinais clínicos. Com base nesses fatos é possível sugerir que o aborto ocorre após a ingestão de doses altas da planta e, pelo menos experimentalmente, em vacas que apresentaram sinais clínicos da intoxicação. Doses menores causam lesões fetais que podem ser responsáveis pelo nascimento de bezerros débeis que podem morrer após o parto. No trabalho mencionado anteriormente, dos três bezerros nascidos com sinais de debilidade dois se recuperaram, os fetos abortados apresentavam autólise avançada e um bezerro que morreu 8 dias após o parto, apresentou necrose hepática (Stolf et al. 1994).

Considerando que o aborto induzido por A. glazioviana é devido à morte fetal causada pelo princípio ativo da mesma, é evidente a necessidade de determinar se a morte do feto ocorre em consequiência das lesões cardíacas, observadas em todos os bezerros, e/ou das lesões do sistema nervoso, observadas no único animal que morreu espontaneamente, ou ainda por outras alterações ainda não estabelecidas. Para isso seria necessário estudar as lesões histológicas em fetos mortos em consequïência da administração de $A$. glazioviana às vacas.

Outras plantas que causam aborto em ruminantes, como Pinus ponderosa, Pinus contorta, Juniperus communis e Cupressus macrocarpa, contêm como princípio ativo ácido isocupréssico que atua diretamente sobre o sistema reprodutivo induzindo abortos por um efeito hormonal, pelo bloqueio da função luteal com diminuição dos níveis de progesterona (Stegelmeier et al. 1996, Wu et al. 2002). Outras plantas abortivas, tais como Gutierrezia spp e Astragalus lentiginosus, possuem princípios ativos que atuam, também, no sistema reprodutivo e/ou endócrino levando ao aborto (Dollahite et al. 1962, Ellis et al. 1985).
Tetrapterys spp causam aborto e lesões cardíacas em ruminantes similares ao que é observado na intoxicação por $A$. glazioviana (Tokarnia et al. 2000), existindo a possibilidade de que seus princípios ativos tenham ação semelhante. A intoxicação experimental por Tetrapterys multiglandulosa em cabras prenhes causa diminuição de batimentos cardíacos nas cabras, morte fetal e abortos (Melo et al. 2001).

As lesões cardíacas observadas na intoxicação por $A$. glazioviana são semelhantes às observadas nas intoxicações por plantas da família Rubiaceae do Sul da África, que contêm como princípio ativo uma substância presente no precipitado etanólico denominada pavetamine (Fourie et al. 1995). O precipitado etanólico extraído de $A$. glazioviana seca a $100^{\circ} \mathrm{C}$ e moída, administrado a um ovino, não causou sinais clínicos de insuficiência cardíaca e tampouco lesões cardíacas macroscópicas ou histológicas (Schild \& Haraguchi 2003, dados não publicados), sugerindo que o princípio ativo da planta poderia ser diferente do das plantas do sul da África, entretanto novos experimentos devem ser realizados para isto ser comprovado.

As lesões macroscópicas observadas no Bezerro 11, caracterizadas por manchas esbranquiçadas discretas na parede do ventrículo direito e vértice do septo interventricular, são descritas na miocardiopatia causada por A. glazioviana, tanto na forma espontânea da intoxicação em bovinos e ovinos (Gava \& Barros 2001, Gava et al. 2003) como na intoxicação experimental nestas espécies (Gava \& Barros 2001, Gava et al. 2001, Stigger et al. 2001, Leite et al. 2002).

As lesões histológicas observadas em dois dos três bezerros que nasceram com sinais clínicos de debilidade caracterizaramse por tumefação e vacuolização multifocal de grupos de cardiomiócitos. No bezerro que nasceu morto, além dessas lesões, foi observada proliferação de fibroblastos entre os feixes de fibras musculares cardíacas, com presença de macrófagos. Essas lesões são similares às observadas em ovinos intoxicados experimentalmente, nos quais descrevem-se lesões histológicas iniciais caracterizadas, também, por tumefação das fibras musculares cardíacas, evidenciadas principalmente nos cortes transversais (Stigger et al. 2001). Em casos mais acentuados os mesmos autores encontraram proliferação fibroblástica e deposição de colágeno nas áreas onde havia desaparecimento completo do sarcoplasma. Em bovinos experimentais descreve-se, também, a presença de grupos multifocais de fibras tumefeitas e vacuolizadas similares ao observado nos Bezerros 11, 12 e 13 deste experimento (Gava et al. 2001). Em casos espontâneos de miocardiopatia em bovinos, causada pela ingestão de $A$. glazioviana, têm sido descritas marcada degeneração e necrose de cardiomiócitos e fibrose acentuada (Gava \& Barros 2001).

No Bezerro 11, cujas lesões cardíacas de fibrose foram mais acentuadas, havia, também, vacuolização discreta no SNC. Espongiose da medula cerebelar, pedúnculos cerebelares, colículos, tálamo, núcleos basais e córtex frontal e parietal tem sido descrita na intoxicação experimental por A. glazioviana em ovinos (Stigger et al. 2001) e na medula cerebelar, pedúnculos cerebelares ponte e medula oblonga na intoxicação natural em bovinos (Gava et al. 2001). É provável que esta lesão seja um fator determinante para a ocorrência de abortos ou natimortos, já que os demais animais deste experimento (Bezerros 12, 13 e 
16), que nasceram a termo, não apresentaram esta alteração, embora clinicamente tenham mostrado sinais de debilidade ao nascimento. Os resultados observados neste experimento não permitam determinar qual a causa do nascimento de bezerros debilitados, se as lesões cardíacas, prováveis lesões do sistema nervoso não observadas na histologia nos animais que não morreram e foram sacrificados ou se outro tipo de lesão não identificada pela histologia.

Aumento do glicogênio foi observado nos cardiomiócitos de todos os animais deste experimento que nasceram com sinais clínicos de intoxicação. Uma sobrecarga do glicogênio celular pode ser consequiência de doenças metabólicas, com alteração no metabolismo dos carboidratos, induzidas por drogas como os corticosteróides; de deficiências enzimáticas como nas doenças de armazenamento hereditárias; nas hiperglicemias de qualquer causa (Cheville 1994). O acúmulo de glicogênio é mais um indicador de consumo celular deficiente que de aumento da atividade metabólica (Ghadyally 1997).

A planta Castanospermum australe possui como princípio ativo a castanospermina, um inibidor da a-glicosidase (Reichmann et al. 1987, Stegelmeier et al. 1995) o qual induz experimentalmente em ratos vacúolos com glicogênio na musculatura esquelética, similares aos da glicogenose hereditária (Stegelmeier et al. 1995), não tendo sido relatado acúmulo de glicogênio no miocárdio. Em bovinos da raça Brahman aos quais foi administrado castanospermina foi observado diminuição da atividade da a-glicosidase (Reichmann et al. 1987).

$\mathrm{O}$ aumento do glicogênio encontrado nos cardiomiócitos dos bezerros desse experimento deve ser o resultado de uma incapacidade das células cardíacas de utilizá-lo adequadamente, possivelmente por interferência nos sistemas enzimáticos responsáveis pela glicogenólise como acontece com a planta Castanospermum australe. $\mathrm{O}$ aumento do glicogênio sugere diminuição da atividade metabólica e da glicogenólise, fortalecendo a hipótese de um dano nos cardiomiócitos induzido pela ação transplacentária de $A$. glazioviana. Não foi possível determinar em que ponto do processo de glicogenólise houve interferência da toxina de $A$. glazioviana. As alterações mitocondriais foram interpretadas como artefatos, pois apareceram, também, no animal controle.

Agradecimentos.- Ao Técnico de Laboratório da UFPel, João Francisco Nunes, pelas preparações histológicas.

\section{REFERÊNCIAS}

Cheville N.F. 1994. Ultrastructural Pathology. An Introduction to Interpretation. Iowa State University Press, Ames, Iowa, p.127-273.

Dollahite J.W., Shaver T. \& Camp B.J. 1962. Injected saponins as abortifacients. Am. J. Vet. Res. 23(97):1261-1263.

Ellis L.C., James L.F., McMullen R.W. \& Panter K.E. 1985. Reduced progesterone and altered cotyledonary prostaglandin values induced by locoweed (Astragalus lentiginosus) in sheep. Am. J. Vet. Res. 46(9):1903190.

Fourie N., Erasmus G.L., Schultz R.A. \& Prozesky L. 1995. Isolation of the toxin responsible for gousiekte, a plant-induced cardiomyopathy of ruminants in southern Africa. Onderstepoort J. Vet. Res. 62:77-87.

Gava A. 1993. Intoxicação por Ateleia glazioviana, p. 222-225. In: RietCorrea F., Méndez M.C. \& Schild A.L. (ed.) Intoxicações por Plantas Tóxi- cas e Micotoxicoses em Animais Domésticos. Editorial Agropecuária, Hemisfério Sur, Montevideo.

Gava A. \& Barros C.S.L. 2001. Field observations of Ateleia glazioviana poisoning in cattle in Southern Brazil. Vet. Human Toxicol. 43(1):37-41.

Gava A., Barros C. S. L., Pilati C., Barros S.S. \& Mori A.M. 2001. Intoxicação por Ateleia glazioviana (Leg.Papilionoideae) em bovinos. Pesq. Vet. Bras. 21(2):49-59.

Gava D. Reis R.N., Rocha T.S., Pasquale E. \& Gava A. 2003. Intoxicação natural por Ateleia glazioviana (Leg.Papilionoideae) em ovinos. XI Encontro Nacional de Patologia Veterinária, Botucatu, SP, 21-25 de julho, p.60.

Ghadially F.N. 1997. Ultrastructural Pathology of the Cell and Matrix. Vol. 2. 4th ed. Butterworth-Heinemann, USA, p.1013-1103.

Leite L.G., Riet-Correa F., Medeiros R.M.T., Piacenti A., Aragão M. \& Shons S. 2002. Susceptibilidade de animais de laboratório à intoxicação experimental por Ateleia glazioviana (Leg.Papilionoideae). Pesq. Vet. Bras. 22(2):73-78

Melo M..M., Vasconcelos A.C., Dantas G.C., Serakides R. \& Alzamora Filho F. 2001. Experimental intoxication of pregnant goats with Tetrapterys multiglandulosa A. Juss. (Malpighiaceae). Arq. Bras. Med. Vet. Zootec. 53(1):58-65.

Reichmann K.G., Twist J.O., McKenzie R.A. \& Rowan K.J. 1987. Inhibition of bovine alpha-glucosidase by Castanospermum australe and its effect on the biochemical identification of heterozygotes for generalised glycogenosis type II (Pompe's disease) in cattle. Aust. Vet. J. 64(9):274-276.

Schultz R.A., Fourie N., Bode M.L., Basson K.M., Labuschagne L., Vleggaar R. \& Medlen C. 2004. Pavetamine: an inhibitor of protein synthesis in the heart, p.408-411. In: Acamovic T, Stewart C.S. \& Pennycott T.W. (ed.) Poisonous Plants and Related Toxins. CABI Publishing, Willingford, UK.

Stegelmeier B.L, Molyneux R.J., Elbein A.D. \& James L.F. 1995. The lesions of locoweed (Astragalus mollisimus), swainsonine, a castanospermine in rats. Vet. Pathol. 32(3):289-298.

Stegelmeier B.L, Gardner D.R., James L.F., Panter K.E. \& Molyneux R.J. 1996. The toxic and abortifacient effects of Ponderosa Pine. Vet. Pathol. 33:22-28

Stigger A.L., Barros C.S.L., Langhor I.M.B. \& Barros S.S. 2001. Intoxicação experimental por Ateleia glazionviana (Leg. Papilionoideae) em ovinos. Pesq. Vet. Bras. 21(3):98-108.

Stolf L., Gava A., Varaschim M.S., Neves D.S., Mondadori A. \& Scolari L.S. 1994. Aborto em bovinos causado pela ingestão de Ateleia glazioviana (Leg,Papilionoidea). Pesq. Vet. Bras. 14:15-18.

Terra F., Riet-Correa G. \& Schild A.L. 2002. Intoxicação experimental por Tetrapterys spp. em ovinos - Reprodução da forma cardíaca. XI Congresso de Iniciação Científica, Universidade Federal de Pelotas, Pelotas. (Resumos)

Tokarnia C.H., Peixoto P.V., Döbereiner J., Consorte L.B. \& Gava, A. 1989. Tetrapterys spp (Malpighiaceae), a causa de mortandades em bovinos caracterizadas por alterações cardíacas. Pesq. Vet. Bras. 9(1-2):23-44.

Tokarnia C.H., Peixoto P.V. \& Döbereiner J. 1990. Poisonous plants affecting heart function of cattle in Brazil. Pesq. Vet. Bras. 10(1-2):1-10.

Tokarnia C.H., Brito M.F., Driemeier D., Costa J.B.D. \& Camargo A.J.R. 1998. Aborto em vacas na intoxicação experimental pelas favas de Styphynodendron obovatum (Leg. Mimosoideae). Pesq. Vet. Bras. 18(1):35-38.

Tokarnia C.H., Döbereiner J., Dutra I.S., Brito I.S., Chagas B.R., França T.N. \& Brust L.A.G. 1999. Experimentos em bovinos com as favas de Enterolobium contortisiliquum e E. timbouva para verificar propriedades fotossensibilizantes e/ou abortivas. Pesq. Vet. Bras. 19(1):39-45.

Tokarnia C.H., Döbereiner J. \& Peixoto P.V. 2000. Plantas Tóxicas do Brasil. Editora Helianthus, Rio de Janeiro, p.19-61.

Wu L.S., Chen J.C., Sheu S.Y., Huang C.C., Kuo Y.H., Chiu C.H., Lian W.X., Yang C.J., Kaphle K. \& Lin J.H. 2002. Isocupressic Acid Blocks Progesterone production from bovine luteal cells. Am. J. Chinese Medicine 30(4):533541. 